\title{
Reconstitución de células natural killer después del trasplante de células progenitoras hematopoyéticas en niños
}

\author{
Israel Parra-Ortega, ${ }^{1}$ Noemí Nájera-Martínez, ${ }^{1}$ Félix Gaytán-Morales, ${ }^{2}$ Iván Castorena-Villa, ${ }^{2}$ \\ Catalina Cortés-Flores, ${ }^{2}$ Briceida López-Martínez, ${ }^{3}$ Vianney Ortiz-Navarrete ${ }^{4}$ e Irlanda Olvera-Gómez ${ }^{5 *}$ \\ ${ }^{1}$ Hospital Infantil de México Federico Gómez, Departamento de Laboratorio Clínico, Ciudad de México; ${ }^{2}$ Hospital Infantil de México Federico Gómez, \\ Unidad de Trasplante de Células Progenitoras Hematopoyéticas, Ciudad de México; ${ }^{3}$ Hospital Infantil de México Federico Gómez, Subdirección \\ de Servicios Auxiliares y Diagnóstico, Ciudad de México; ${ }^{4}$ Centro de Investigación y de Estudios Avanzados, Biomedicina Molecular, Ciudad de \\ México; 5Universidad Anáhuac Norte, Estado de México. México
}

\section{Resumen}

Introducción: Después de un trasplante de células progenitoras hematopoyéticas (TCPH), la reconstitución de las células natural killer (NK) es la principal barrera contra las infecciones virales. Objetivo: Determinar que el conocimiento sobre la cinética de la reconstitución de las células NK posterior al TCPH contribuye a un eficiente monitoreo del trasplante, lo que incrementa la posibilidad de éxito de este. Método: Se incluyeron 21 pacientes sometidos a TCPH, así como un grupo control de individuos clínicamente sanos. En diferentes momentos después del trasplante (intervalo de 21 a 670 días), mediante citometría de flujo se cuantificaron las células NK CD3-CD16+CD56+ en muestras de sangre periférica. Resultados: La recuperación de las células NK ocurre entre los tres y seis meses y entre los 10 y 12 meses postrasplante; su número fue significativamente menor (en comparación con el grupo control) en el tiempo restante del monitoreo. Conclusiones: El primer periodo de recuperación de las células NK ocurre entre los tres y seis meses posteriores al trasplante. La reconstitución es transitoria y el número de células NK varía en los primeros años.

PALABRAS CLAVE: Células natural killer. Trasplante. Células hematopoyéticas. Reconstitución celular.

\section{Natural killer cell reconstitution after hematopoietic stem-cell transplantation in children}

\begin{abstract}
Introduction: After hematopoietic stem cell transplantation (HSCT), natural killer (NK) cells reconstitution is the main barrier against viral infections. Objective: To determine that the knowledge on the kinetics of NK cell reconstitution after HSCT contributes to transplant efficient monitoring, which increases the possibility of its success. Method: Twenty-one patients undergoing HSCT were included, as well as a control group of clinically healthy individuals. At different time points after transplantation (range of 21 to 670 days), CD3- CD16+ CD56+ NK cells were quantified by flow cytometry in peripheral blood samples. Results: NK cell recovery occurs at three to six months and 10 to 12 months post-transplantation; their number was significantly lower (in comparison with the control group) in the rest of the monitoring time. Conclusions: The first period of NK cell recovery occurs between three and six months after transplantation. Reconstitution is transient and the number of NK cells varies in the first years.
\end{abstract}

KEY WORDS: Natural killer cells. Transplantation. Hematopoietic cells. Cell reconstitution. 


\section{Introducción}

Las células natural killer (NK) se generan a partir de un precursor linfoide proveniente de células progenitoras de médula ósea. ${ }^{1,2}$ Su morfología es similar a la de los linfocitos $T$ y $B$, pero son profusamente granuladas y se identifican por la ausencia de CD3 (molécula exclusiva de los linfocitos T) y la expresión de CD16 y CD56 (más de $90 \%$ de las células NK en sangre periférica expresan ambas), si bien existen subpoblaciones con los fenotipos CD16- CD56+ y CD16+ CD56-, que constituyen entre 5 y $10 \%$ del total de este tipo de células. ${ }^{3}$ Las NK son la primera barrera de defensa contra los patógenos (principalmente virus) y las células tumorales. Su activación e inhibición acontece tras el reconocimiento de alelos del antígeno de leucocitos humanos (human leukocyte antigen, $\mathrm{HLA}$ ) a través de receptores similares a las inmunoglobulinas que inhiben a las células asesinas, los cuales pueden interaccionar con un único alelo del HLA o con varios. ${ }^{4}$

Independientemente de la fuente de injerto (médula ósea, sangre periférica movilizada o células de cordón umbilical), la reconstitución de la población de células NK se alcanza después de uno a dos meses del trasplante de células progenitoras hematopoyéticas (TCPH) de origen alogénico. En general, las células NK que se generan durante este periodo no derivan de la expansión de las células NK maduras del injerto, sino principalmente de la diferenciación y maduración de las células progenitoras. ${ }^{5-8}$

Además, en los trasplantes alogénicos se puede producir alorreactividad de las células ${ }^{9-11}$ provenientes del injerto (células maduras) o de las generadas en el receptor después del TCPH, evento que puede tener un efecto citotóxico sobre las células tumorales que resistieron o reaparecieron después del TCPH. ${ }^{12}$

La reconstitución de las células de la inmunidad innata es crítica en los pacientes con inmunosupresión, así como en los receptores de TCPH (alogénico o autólogo), debido a su papel en el control de las infecciones. En el último caso, dado que la reconstitución de las células $T$ y $B$ requiere al menos seis meses, es relevante la pronta recuperación de las estirpes celulares asociadas al control de los patógenos, como las células NK.

La reconstitución de las células NK posterior al TCPH requiere tiempo para alcanzar la homeostasis de esta estirpe celular en los primeros años posteriores al trasplante alogénico. El monitoreo de este proceso contribuirá a una valoración integral del receptor de un trasplante, al incluir exámenes de laboratorio y seguimiento clínico, con la finalidad de aumentar la posibilidad de éxito del procedimiento.

\section{Método}

Se realizó un estudio observacional, prospectivo y descriptivo de niños sometidos a TCPH de tipo alogénico entre enero de 2017 y enero de 2018 en la Unidad de Trasplante de Células Progenitoras y el Laboratorio Clínico del Hospital Infantil de México "Federico Gómez". El protocolo fue autorizado por los Comités de Investigación, Ética y Bioseguridad del hospital. Los padres, tutores o cuidadores otorgaron por escrito su consentimiento previa información del objetivo de la investigación.

Los pacientes recibieron un régimen de acondicionamiento mieloablativo o de intensidad reducida (de acuerdo a la patología de base) y posteriormente se infundió al menos una dosis de $2 \times 10^{6} \mathrm{CD} 34+/ \mathrm{kg}$ de peso corporal. Se incluyó un grupo control $(n=20)$ integrado por individuos clínicamente sanos, sin fiebre, diagnóstico de infección ni en tratamiento con antibióticos o antivirales en los últimos cinco días.

Desde el día 21 hasta el día 670 de la etapa postrasplante, de cada individuo se recolectó aproximadamente $1 \mathrm{~mL}$ de sangre periférica. La biometría hemática se realizó en equipo Coulter LH 780® (Beckman Coulter, Brea, CA, Estados Unidos) para determinar el número de leucocitos totales, así como el porcentaje de linfocitos del individuo. Se realizó la fenotipificación y cuantificación de las células NK (CD3- CD16+ CD56+) mediante citometría de flujo; se analizaron al menos 50000 células. Se realizaron las determinaciones y se calcularon los valores absolutos (número de células por litro) de células NK. Además, se recolectaron datos de la genotipificación del HLA obtenido previamente en el Laboratorio de Histocompatibilidad.

\section{Resultados}

Durante el periodo de enero de 2017 y enero de 2018 se incluyeron 21 pacientes sometidos a TCPH. Los diagnósticos por los cuales fueron referidos a la Unidad de Trasplante de Células Progenitoras Hematopoyéticas fueron los siguientes: leucemia linfoblástica aguda, $38.09 \%(n=8)$; aplasia medular, $28.57 \%(n=6)$; leucemia mieloide aguda, $19.04 \%$ $(n=4)$; otros tumores sólidos, $14.28 \%(n=3)$ 


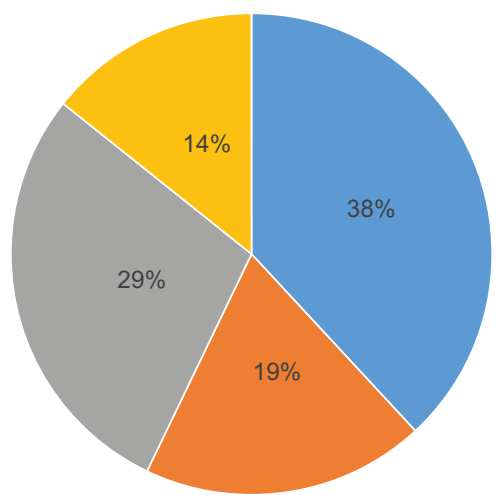

- Leucemia aguda linfoblástica - Leucemia aguda mieloide

- Aplasia medular

-Otros (tumores sólidos)

Figura 1. Diagnósticos por los que se realizó trasplante de células progenitoras hematopoyéticas.

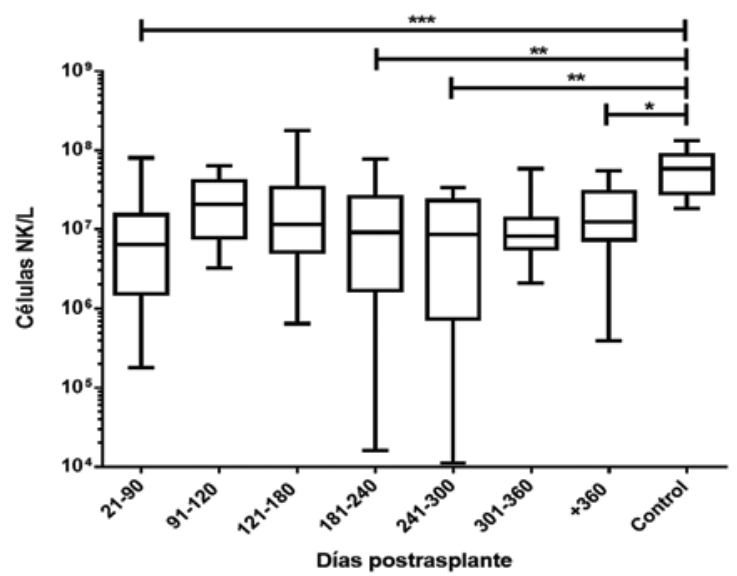

Figura 2. Cinética de la reconstitución de las células NK después del trasplante de células progenitoras hematopoyéticas. Los valores se transformaron a logaritmos y se presentan los valores de $p$ conforme la prueba de Kruskal-Wallis ( $p<0.0001)$ y la comparación múltiple. ${ }^{*} p=0.043,{ }^{* *} p=0.0059,{ }^{* * *} p<0.0001$.

(Figura 1); nueve pacientes (43\%) fueron del sexo masculino y 12 (57\%), del femenino; la edad osciló entre uno y 17 años (Tabla 1).

Algunos pacientes recibieron un régimen de acondicionamiento mieloablativo y otros de intensidad reducida (según la patología de base) antes del trasplante de células hematopoyéticas alogénicas provenientes de sangre periférica, posterior a la movilización con factor estimulante de colonias granulocíticas en el donador. La compatibilidad entre el donador y el receptor fue definida con base en la genotipificación de los alelos HLA-A, HLA-B, HLA-C y HLA-DP en dos pacientes y en los 19 restantes además se analizaron
Tabla 1. Características demográficas de los pacientes sometidos a TCPH y de los sujetos clínicamente sanos del grupo control

Datos demográticos Pacientes con TCPH Individuos control

Sexo

\begin{tabular}{l|l|l}
\hline Masculino & $9(43 \%)$ & $17(85 \%)$
\end{tabular}

\begin{tabular}{l|l|l}
\hline Femenino & $12(57 \%)$ & $3(15 \%)$
\end{tabular}

Edad

$\begin{array}{lll}\text { Intervalo } & 1 \text { a } 17 \text { años } & 8 \text { a } 17 \text { años }\end{array}$

Media $\quad 7$ años 6 meses 10 años 1 mes

\begin{tabular}{l|l} 
Mediana & 8 años 5 meses
\end{tabular}

$\mathrm{TCPH}=$ trasplante de células progenitoras hematopoyéticas.

HLA-DQ y HLA-DR. En la Tabla 2 se indican los alelos compartidos y si se trató de un trasplante haploidéntico cuando el donador fue uno de los padres.

Para analizar la cinética de reconstitución de las células NK en los 21 pacientes se realizaron 105 determinaciones, con un promedio de cinco por paciente (mínimo dos y máximo 11). Los valores obtenidos se agruparon por periodos: de 21 a 90 días, de 91 a 120 días, de 121 a 180 días, de 181 a 240 días, de 241 a 300 días, de 301 a 360 días y más de 360 . Todas las categorías se compararon con los valores obtenidos en los individuos clínicamente sanos (Tabla 3 y Figura 2). El origen de las células identificadas en las primeras semanas podría deberse a la expansión de las células incluidas en el injerto (dado que no se realiza un enriquecimiento de las células CD34+), en tanto que las detectadas posteriormente serían las generadas en el receptor a partir de precursores celulares, principalmente del donador, como lo demuestra el $95 \%$ de quimerismo detectado en células de sangre periférica (Figura 3).

Se observaron diferencias significativas respecto a los números absolutos de células NK en los pacientes sometidos a TCPH: durante todo el periodo de estudio fueron inferiores a los del grupo control (Tabla 3 y Figura 3).

\section{Discusión}

De acuerdo con lo observado en los pacientes analizados, la recuperación temprana de las células NK acontece en el periodo de tres a seis meses postrasplante; sin embargo, se observan momentos en los que el número absoluto es menor, por lo que diversos eventos como infecciones y enfermedad injerto contra hospedero (EICH) podrían afectar negativamente a la reconstitución de las células NK. 
Tabla 2. Dosis celular del trasplante, régimen de acondicionamiento y compatibilidad del HLA

\begin{tabular}{|c|c|c|c|}
\hline Paciente & Dosis celular CD34+/kg & Régimen de acondicionamiento & Compatibilidad de alelos del HLA* \\
\hline 1 & $1.71 \times 10^{7}$ & Fludarabina/ciclofosfamida & Haploidéntico \\
\hline 2 & $7.4 \times 10^{6}$ & Busulfán/ciclofosfamida & Alogénico (12/12) \\
\hline 3 & $6.7 \times 10^{6}$ & Ciclofosfamida/etopósido/radioterapia & Alogénico (8/8) \\
\hline 4 & $3.19 \times 10^{6}$ & Busulfán/ciclofosfamida & Alogénico (12/12) \\
\hline 5 & $5 \times 10^{6}$ & Busulfán/ciclofosfamida & Alogénico (12/12) \\
\hline 6 & $1.03 \times 10^{7}$ & Busulfán/fludarabina & Haploidéntico \\
\hline 7 & $4 \times 10^{6}$ & Busulfán/ciclofosfamida & Alogénico (12/12) \\
\hline 8 & $8 \times 10^{6}$ & Busulfán/ciclofosfamida/radioterapia & Haploidéntico \\
\hline 9 & $7.09 \times 10^{6}$ & Fludarabina/ciclofosfamida & Haploidéntico \\
\hline 10 & $1.47 \times 10^{6}$ & Busulfán/ciclofosfamida/radioterapia & Alogénico (8/8) \\
\hline 11 & $5 \times 10^{6}$ & Busulfán/ciclofosfamida & Alogénico (12/12) \\
\hline 12 & $1.11 \times 10^{6}$ & Busulfán/ciclofosfamida & Alogénico (12/12) \\
\hline 13 & $1.73 \times 10^{6}$ & Busulfán/ciclofosfamida & Alogénico (12/12) \\
\hline 14 & $2.52 \times 10^{6}$ & Busulfán/ciclofosfamida & Alogénico (12/12) \\
\hline 15 & $1.54 \times 10^{5}$ & Busulfán/ciclofosfamida & Alogénico (11/12) \\
\hline 16 & $6 \times 10^{6}$ & Fludarabina/ciclofosfamida & Haploidéntico \\
\hline 17 & $6 \times 10^{6}$ & Busulfán/ciclofosfamida/radioterapia & Alogénico (12/12) \\
\hline 18 & $1.23 \times 10^{6}$ & Busulfán/ciclofosfamida & Alogénico (12/12) \\
\hline 19 & $1 \times 10^{6}$ & Busulfán/ciclofosfamida & Alogénico (12/12) \\
\hline 20 & $9.92 \times 10^{6}$ & Fludarabina/ciclofosfamida & Haploidéntico \\
\hline 21 & $4.8 \times 10^{6}$ & Busulfán/ciclofosfamida & Alogénico (11/12) \\
\hline
\end{tabular}

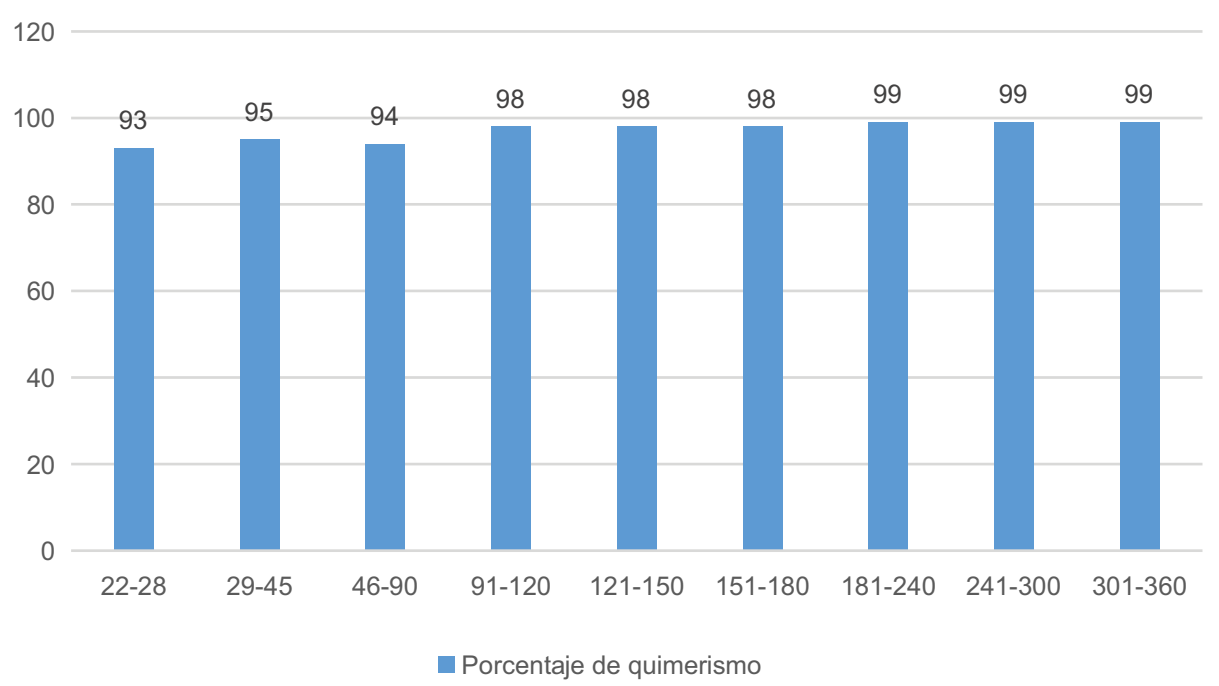

Figura 3. Cinética del quimerismo celular posterior al trasplante de células progenitoras hematopoyéticas. Media obtenida en los diferentes tiempos postrasplante. 
Tabla 3. Valores de las mediciones de células NK/L en las diferentes determinaciones realizadas

\begin{tabular}{|c|c|c|c|c|c|c|c|c|}
\hline \multirow{2}{*}{$\begin{array}{l}\text { Valor } \\
\text { estadístico }\end{array}$} & \multicolumn{7}{|c|}{ Días postrasplante } & \multirow{2}{*}{$\begin{array}{l}\text { Grupo } \\
\text { control }\end{array}$} \\
\hline & $21-90$ & $91-120$ & $121-180$ & $180-240$ & $241-300$ & $301-360$ & +360 & \\
\hline Valor mínimo & $0.018 \times 10^{8}$ & $0.0321 \times 10^{8}$ & $0.0065 \times 10^{8}$ & $0.00016 \times 10^{8}$ & $0.000011 \times 10^{8}$ & $0.0208 \times 10^{8}$ & $0.00040 \times 10^{8}$ & $0.183 \times 10^{8}$ \\
\hline Percentil 25 & $0.161 \times 10^{8}$ & $0.0778 \times 10^{8}$ & $0.051 \times 10^{8}$ & $0.0167 \times 10^{8}$ & $0.00743 \times 10^{8}$ & $0.0563 \times 10^{8}$ & $0.0730 \times 10^{8}$ & $0.283 \times 10^{8}$ \\
\hline Mediana & $0.635 \times 10^{8}$ & $0.205 \times 10^{8}$ & $0.0115 \times 10^{8}$ & $0.0909 \times 10^{8}$ & $0.0854 \times 10^{8}$ & $0.0819 \times 10^{8}$ & $0.124 \times 10^{8}$ & $0.582 \times 10^{8}$ \\
\hline Percentil 75 & $0.144 \times 10^{8}$ & $0.406 \times 10^{8}$ & $0.0335 \times 10^{8}$ & $0.258 \times 10^{8}$ & $0.231 \times 10^{8}$ & $0.135 \times 10^{8}$ & $0.295 \times 10^{8}$ & $0.874 \times 10^{8}$ \\
\hline $\begin{array}{l}\text { Valor } \\
\text { máximo }\end{array}$ & $0.794 \times 10^{8}$ & $0.633 \times 10^{8}$ & $1.76 \times 10^{8}$ & $0.765 \times 10^{8}$ & $0.335 \times 10^{8}$ & $0.5 .82 \times 10^{8}$ & $0.548 \times 10^{8}$ & $1.31 \times 10^{8}$ \\
\hline Media & $0.114 \times 10^{8}$ & $0.252 \times 10^{8}$ & $0.289 \times 10^{8}$ & $0.165 \times 10^{8}$ & $0.126 \times 10^{8}$ & $0.1 .47 \times 10^{8}$ & $0.183 \times 10^{8}$ & $0.605 \times 10^{8}$ \\
\hline $\begin{array}{l}\text { Desviación } \\
\text { estándar }\end{array}$ & $0.160 \times 10^{8}$ & $0.203 \times 10^{8}$ & $0.442 \times 10^{8}$ & $0.232 \times 10^{8}$ & $0.126 \times 10^{8}$ & $0.1 .95 \times 10^{8}$ & $0.148 \times 10^{8}$ & $0.334 \times 10^{8}$ \\
\hline $\begin{array}{l}\text { Error } \\
\text { estándar }\end{array}$ & $0.0296 \times 10^{8}$ & $0.0642 \times 10^{8}$ & $0.111 \times 10^{8}$ & $0.067 \times 10^{8}$ & $0.0419 \times 10^{8}$ & $0.074 \times 10^{8}$ & $0.0316 \times 10^{8}$ & $0.0927 \times 10^{8}$ \\
\hline $\begin{array}{l}\text { IC a } 95 \% \\
\text { límite inferior }\end{array}$ & $0.0530 \times 10^{8}$ & $0.107 \times 10^{8}$ & $0.0531 \times 10^{8}$ & $0.0172 \times 10^{8}$ & $0.0292 \times 10^{8}$ & $0.034 \times 10^{8}$ & $0.118 \times 10^{8}$ & $0.403 \times 10^{8}$ \\
\hline $\begin{array}{l}\text { IC a } 95 \% \\
\text { límite superior }\end{array}$ & $0.174 \times 10^{8}$ & $0.398 \times 10^{8}$ & $0.525 \times 10^{8}$ & $0.312 \times 10^{8}$ & $0.223 \times 10^{8}$ & $0.327 \times 10^{8}$ & $0.249 \times 10^{8}$ & $0.807 \times 10^{8}$ \\
\hline $\begin{array}{l}\text { Comparación } \\
\text { con el grupo } \\
\text { control, } \\
\text { diferencia (p) }\end{array}$ & $\begin{array}{c}\text { Sí } \\
(p<0.0001)\end{array}$ & No & No & $\begin{array}{c}\text { Sí } \\
(p=0.0059)\end{array}$ & $\begin{array}{c}\text { Sí } \\
(p=0.0059)\end{array}$ & No & $\begin{array}{c}\text { Sí } \\
(p=0.043)\end{array}$ & - \\
\hline
\end{tabular}

No se encontró diferencia significativa al analizar el número de células NK en 36 muestras de pacientes con $\mathrm{EICH}$ versus 69 muestras de pacientes sin $\mathrm{EICH}$ (datos no mostrados), ni tampoco al comparar las cuantificaciones de muestras que presentaban o no agentes virales: poliomavirus BK, citomegalovirus, adenovirus, Epstein-Barr (datos no mostrados), por lo que se consideró que la reconstitución de células NK en este grupo de pacientes no fue afectada significativamente por esas entidades clínicas.

Lo anterior podría deberse a que en los cuatro pacientes en quienes se detectó ADN viral del poliomavirus $B K$ se redujo el tratamiento inmunosupresor con la finalidad de permitir la función eficiente de las células (expansión y capacidad efectora, como la producción de interferón-gamma y la citotoxicidad). Cuando el ADN viral detectado fue del virus EpsteinBarr, citomegalovirus o adenovirus (carga viral inferior a 1000 copias $/ \mathrm{mL}$ de plasma) se continuó con el tratamiento estándar y el monitoreo constante de las cargas virales, pues se pretendía que hubiera un control de la infección por el propio receptor. Con el propósito de reducir la severidad de la $\mathrm{EICH}$ se intensificó el tratamiento inmunosupresor, medida que también se realiza cuando el número de células NK se encuentran dramáticamente disminuidas, para favorecer la proliferación de dichas células.

Se ha descrito que la recuperación de las células NK en los pacientes sometidos a TCPH acontece en los primeros dos meses, ${ }^{8,13-15}$ pero que puede retrasarse hasta seis meses en los pacientes con infecciones (principalmente fúngicas) en los primeros 100 días posteriores al trasplante. ${ }^{15}$ Este fenómeno se observó en los pacientes analizados, en quienes se documentaron diversos eventos adversos (infecciones y EICH) durante los 100 primeros días (se identificó virus Epstein-Barr, citomegalovirus, adenovirus en cuatro pacientes y EICH en dos, quienes a lo largo del primer año no tuvieron una reconstitución eficiente de células NK).

Según la literatura, durante el primer mes de la etapa postrasplante el número de células NK debe ser $>0.75 \times 10^{8} / \mathrm{L}$ cuando la fuente de obtención de las células CD34+ es la médula ósea; cuando las células CD34+ son recolectadas de sangre periférica, la reconstitución se obtiene a partir de los cuatro meses en pacientes pediátricos. ${ }^{16}$ En una serie de 
pacientes se logró identificar que la reconstitución alcanzó una mediana de 305 células NK/ $\mu \mathrm{L}$ (rango de $30 \pm 1200$ ) para el día 130 en promedio. ${ }^{7}$ Los anteriores valores son superiores a los obtenidos en los pacientes y el grupo control de la investigación aquí presentada, por lo que la comparación numérica entre las diferentes series no es pertinente dadas las diferentes características de cada grupo, en las cuales influyen diversos factores externos:

- Enfermedad por la que se realiza el TCPH.

- Esquema de acondicionamiento (el cual aunado a la administración de $50 \mathrm{mg} / \mathrm{kg} /$ día de ciclofosfamida en los días 3 y 4 postrasplante, como medida profiláctica para la prevención de $\mathrm{EICH}$ agudo, generó una disminución de la linfopoyesis en nuestros pacientes $y$, por ende, la recuperación de las células NK fue afectada durante los primeros 90 días de la etapa postrasplante).

- Tipo de trasplante.

- Tipo de donador y compatibilidad de HLA (13 TCPH de la serie aquí descrita [62 \%] fueron de tipo alogénico con donador $100 \%$ compatible, dos TCPH [9.5\%] fueron de tipo alogénico con donador $90 \%$ compatible y seis [28.5\%] fueron de tipo haploidéntico).

Al comparar el número de células NK en los pacientes sometidos a TCPH versus el de los individuos control se observó diferencia significativa en algunos periodos (Figura 2), lo cual evidencia que la reconstitución fue transitoria y deficiente en algunos casos, probablemente debido al secuestro de células NK en el sitio blanco de la infección (es decir, vías urinarias en el paciente en quien se identificó virus BK) o a la migración de células a tejidos específicos en los casos de EICH (tejido cutáneo y hepático). El mayor número de células NK en la etapa postrasplante se observó en las cuantificaciones realizadas entre los 91 y 120 días, con una distribución homogénea, de lo que se infiere que existió reconstitución retrasada de células NK en los pacientes, considerando los datos reportados en la literatura. Otro factor que influye en la cuantificación de células NK es la inmunomodulación en la etapa postrasplante, que se realiza como parte del seguimiento al mantenimiento del trasplante.

Existen dos factores que influyen directamente en el retraso de la reconstitución inmunitaria después del TCPH:

- El daño celular en el microambiente generado por el régimen de acondicionamiento.

- La lenta generación de novo de células del sistema inmunitario derivadas del donador, ${ }^{7,17}$ por lo que una de las estrategias para favorecer una reconstitución inmune eficiente es la protección del microambiente del receptor. ${ }^{18}$

En la presente investigación únicamente se analizaron los números absolutos y porcentajes de células NK, sin embargo, la homeostasis del sistema inmune depende también de la capacidad funcional de las mismas, ya que las capacidades efectoras de las células NK pueden ser eficientes en la producción de citocinas, anticuerpos o capacidad citotóxica aun sin que se alcancen los valores observados en los sujetos sanos.

En los receptores de TCPH, quienes se encuentran inmunosuprimidos, ocurren eventos de estimulación antigénica debido a infecciones virales o bacterianas oportunistas; por lo tanto, la participación de las células NK es fundamental al ser la primera línea de defensa inmunológica y su reconstitución es importante para el éxito del trasplante a largo plazo. ${ }^{19-21}$

La evaluación de la función de las diferentes poblaciones de células NK en los receptores de células progenitoras hematopoyéticas puede ayudar al diseño de nuevas intervenciones terapéuticas dirigidas a potenciar los efectos del TCPH..$^{19,22}$

\section{Conflicto de intereses}

\section{Ninguno.}

\section{Fuente de financiamiento}

Este trabajo fue financiado con fondos de los proyectos HIM2017-038 y HIM2017-130FF.

\section{Responsabilidades éticas}

Los autores declaran que para esta investigación no se realizaron experimentos en seres humanos ni en animales.

Confidencialidad de los datos. Los autores declaran que siguieron los protocolos de su centro de trabajo sobre la publicación de datos de terceros.

Derecho a la privacidad y consentimiento informado. Los autores han obtenido el consentimiento informado de los pacientes referidos en el artículo. Este documento obra en poder del autor de correspondencia.

\section{Bibliografía}

1. Lanier LL, Phillips JH, Hackett J, Tutt M, Kumar V. Natural killer cells: definition of a cell type rather than a function. J Immunol. 1986;137:27352739 . 
2. Vivier E, Ugolini S. Natural killer cells: from basic research to treatments. Front Immunol. 2011;2:18

3. Moretta A, Marcenaro E, Parolini S, Ferlazzo G, Moretta L. NK cells a the interface between innate and adaptive immunity. Cell Death Differ 2008:15:226-233.

4. Lanier LL. Up on the tightrope: natural killer cell activation and inhibition. Nat Immunol. 2008;9:495-502.

5. Huenecke S, Cappel C, Esser R, Pfirrmann V, Salzmann-Manrique E, Betz S, et al. Development of three different NK cell subpopulations during immune reconstitution after pediatric allogeneic hematopoietic stem cell transplantation: prognostic markers in GvHD and viral infections. Front Immunol. 2017:8:109.

6. Ding Y, Zhou L, Xia Y, Wang W, Wang Y, Li L, et al. Reference values for peripheral blood lymphocyte subsets of healthy children in China. J Allergy Clin Immunol. 2018:142:970-973.

7. Eyrich $\mathrm{M}$, Lang $\mathrm{P}$, Lal S, Bader $\mathrm{P}$, Handgretinger R, Klingebiel T, et al. A prospective analysis of the pattern of immune reconstitution in a paediatric cohort following transplantation of positively selected human leucocyte antigen-disparate haematopoietic stem cells from parental donors. Br J Haematol. 2001;114:422-432

8. Hokland M, Jacobsen N, Ellegaard J, Hokland P. Natural killer function following allogeneic bone marrow transplantation. Very early reemergence but strong dependence of cytomegalovirus infection. Transplantation. 1988;45:1080-1084.

9. Bellone G, Valiante NM, Viale O, Ciccone E, Moretta L, Trinchieri G. Regulation of hematopoiesis in vitro by alloreactive natural killer cell clones. J Exp Med. 1993;177:1117-1125.

10. Manjili MH. The adaptation model of immunity. Immunotherapy. 2014; 6:59-70.

11. Ciccone E, Pende D, Viale O, Di Donato C, Tripodi G, Orengo AM, et al. Evidence of a natural killer (NK) cell repertoire for (allo) antigen recognition: definition of five distinct NK-determined allospecificities in humans. J Exp Med. 1992;175:709-718.

12. Ruggeri L, Capanni M, Casucci M, Volpi I, Tosti A, Perruccio K, et al. Role of natural killer cell alloreactivity in HLA-mismatched hematopoietic stem cell transplantation. Blood. 1999;94:333-339.
13. Saliba RM, Rezvani K, Leen A, Jorgensen J, Shah N, Hosing C, et al. General and virus-specific immune cell reconstitution after double cord blood transplantation. Biol Blood Marrow Transplant. 2015;21: 1284-1290.

14. Petersen SL, Ryder LP, Björk P, Madsen HO, Heilmann C, Jacobsen N, et al. A comparison of T-, B- and NK-cell reconstitution following conventional or nonmyeloablative conditioning and transplantation with bone marrow or peripheral blood stem cells from human leucocyte antigen identical sibling donors. Bone Marrow Transplant. 2003;32:65-72.

15. Orange JS. Human natural killer cell deficiencies. Curr Opin Allergy Clin mmunol. 2006;6:399-409.

16. De Koning C, Plantinga M, Besseling P, Boelens JJ, Nierkens S. Immune reconstitution after allogeneic hematopoietic cell transplantation in children. Biol Blood Marrow Transplant. 2016;22:195-206.

17. Huttunen $P$ Taskinen $M$, Siitonen $S$, Saarinen-Pinkala UM Impact of very early $\mathrm{CD}^{+} / \mathrm{CD} 8^{+} \mathrm{T}$ cell counts on the occurrence of acute graftversus-host disease and NK cell counts on outcome after pediatric allogeneic hematopoietic stem cell transplantation. Pediatr Blood Cancer. 2015;62:522-528.

18. Mehta RS, Rezvani K. Immune reconstitution post allogeneic transplant and the impact of immune recovery on the risk of infection. Virulence. 2016;7:901-916.

19. Jacobs R, Stoll M, Stratmann G, Leo R, Link H, Schmidt RE. CD16- CD56+ natural killer cells after bone marrow transplantation. Blood. 1992;79: 3239-344.

20. Nguyen S, Kuentz M, Vernant JP, Dhedin N, Bories D, Debré P, et al. Involvement of mature donor T cells in the NK cell reconstitution after haploidentical hematopoietic stem-cell transplantation. Leukemia. 2008;22:344-352.

21. Kheav VD, Busson M, Scieux C, Peffault-de Latour R, Maki G, Haas P, et al. Favorable impact of natural killer cell reconstitution on chronic graft-versus-host disease and cytomegalovirus reactivation after allogeneic hematopoietic stem cell transplantation. Haematologica. 2014;99: 1860-1867.

22. Stabile H, Fionda C, Gismondi A, Santoni A. Role of distinct natural killer cell subsets in anticancer response. Front Immunol. 2017;8:293. 